\title{
Danio rerio: Small Fish Making a Big Splash in Leukemia
}

\author{
Barbara Squiban · J. Kimble Frazer
}

Published online: 18 March 2014

(c) Springer Science+Business Media New York 2014

\begin{abstract}
Zebrafish (Danio rerio) are widely used for developmental biology studies. In the past decade, $D$. rerio have become an important oncology model as well. Leukemia is one type of cancer where zebrafish are particularly valuable. As vertebrates, fish have great anatomic and biologic similarity to humans, including their hematopoietic and immune systems. As an experimental platform, $D$. rerio offer many advantages that mammalian models lack. These include their ease of genetic manipulation, capacity for imaging, and suitability for large-scale phenotypic and drug screens. In this review, we present examples of these strategies and others to illustrate how zebrafish have been and can be used to study leukemia. Besides appraising the techniques researchers apply and introducing the leukemia models they have created, we also highlight recent and exciting discoveries made using $D$. rerio with an eye to where the field is likely headed.
\end{abstract}

Keywords Zebrafish · Model for pathobiology · Leukemia $\cdot$ Transgenesis · Transplantation · Drug Screen

B. Squiban

Section of Pediatric Hematology/Oncology, Department of Pediatrics, University of Oklahoma Health Sciences Center, 941 Stanton L. Young Blvd., BSEB 229, Oklahoma City, OK 73104, USA

e-mail: barbara-squiban@ouhsc.edu

J. K. Frazer $(\square)$

Section of Pediatric Hematology/Oncology, Department of Pediatrics, University of Oklahoma Health Sciences Center, 941 Stanton L. Young Blvd., BSEB 224, Oklahoma City, OK 73104, USA

e-mail: kimble-frazer@ouhsc.edu

\section{Abbreviations}

ALL Acute lymphoblastic leukemia

AML Acute myeloid leukemia

CLL Chronic lymphocytic leukemia

CML Chronic myelogenous leukemia

dpf Days post-fertilization

ENU $\quad N$-ethyl- $N$-nitrosourea

GFP Green fluorescent protein

GOF Gain of function

HSC Hematopoietic stem cell

HTS High-throughput sequencing

IHC Immunohistochemistry

ISH In situ hybridization

LIC Leukemia-initiating cell

LOF Loss of function

MDS Myelodysplastic syndrome

MPD Myeloproliferative disorder

PGE2 Prostaglandin E2

RGENs RNA-guided endonucleases

TALENs Transcription activator-like effector nucleases

T-ALL T cell ALL

TILLING Targeting induced local lesions in genomes

ZFNs Zinc finger nucleases

\section{Introduction}

Leukemias are cancers of blood cells or their precursors, and the word leukemia derives from the Ancient Greek (leukos $=$ white, haima $=$ blood $)$. Accordingly, most leukemias are malignancies of true white blood cells (leukocytes): lymphocytes, monocytes, or other myeloid cells. However, rare leukemias of red blood cell (erythroblastic) and platelet (megakaryoblastic) precursors can also occur. Both the National Cancer Institute of the US and Cancer 
Research UK list leukemia among the 12 most common cancers in their registries [1, 2], and in children and adolescents, leukemia is the most common malignancy, accounting for $>30 \%$ of all cases [3].

There are many types of leukemia, but most cases can be classified based on their rate of progression, acute versus chronic, and the original cell type that is transformed, myeloid vs. lymphoid. Thus, there are four primary categories of leukemias: acute myeloid leukemia (AML), chronic myelogenous leukemia (CML), acute lymphoblastic leukemia (ALL), and chronic lymphocytic leukemia (CLL ). Confusingly, myeloid, myelogenous, myelocytic, and myeloblastic are often used interchangeably; all refer to the same disease. Likewise, lymphocytic, lymphoid, and lymphoblastic all refer to leukemias of lymphocytes.

In recent years, great progress has been made in treating these diseases. Many forms of leukemia, in particular pediatric pre-B cell ALL, are now highly curable [4]. This success has been accompanied-and often driven-by improved understanding of molecular mechanisms fostering neoplasia. Besides conceptualizing why cancers occur, discoveries of oncogenic drivers can also reveal potential therapeutic targets. A noteworthy example is the identification of $\mathrm{ABL}$ kinase inhibition as a highly efficacious treatment in CML, a cancer that harbors $B C R-A B L$ translocations in nearly all cases [5, 6].

To advance our knowledge of leukemogenesis and realize goals of "molecularly tailored therapy" and "personalized medicine" as foreshadowed by ABL inhibitors in CML, it is vital that we learn the key oncogenes, tumor suppressors, and genetic pathways operative in less homogeneous leukemias than CML. In addition, even in leukemias where key molecular drivers are known, much work remains to find more effective and less toxic drugs and to develop simpler and shorter treatment regimens. Finally, the multigenic nature of leukemias and their complex organismal-environmental interactions leave us lacking with regard to lofty ambitions such as blocking cancer initiation and employing chemo-prevention strategies.

Clinical samples, human cell lines, and murine models are the mainstays for studies of leukemia, but simpler metazoans such as Caenorhabditis elegans [7] and Drosophila melanogaster [8-11] have also enhanced our understanding of oncogenesis. Zebrafish (Danio rerio) represent an intermediate between such models and mice, preserving many experimental advantages of invertebrates, yet also conserving key vertebrate anatomic features and human cell types. In particular, D. rerio are suitable for leukemia studies, because fish share crucial hematopoietic organs, tissues, and cells with mammals. Notably, zebrafish possess blood-forming marrow, a spleen and thymuswhich exist only in jawed vertebrates-and the cells where most human leukemias arise, such as B and T lymphocytes
Fig. 1 Development and applications of zebrafish leukemia models. D. rerio leukemia models can be created via unbiased forward genetic screens, using reverse strategies to dampen/mis-express/mutate genes or introduce transgenes, or by transplanting leukemic cells into fish recipients. Once a model has been engineered, it can be investigated in several ways. Genetic and molecular biology techniques can identify new genes of interest or test candidates in known oncogenic pathways. Mechanisms of leukemogenesis, progression, treatment resistance, or other key biology can be probed in vivo. Fish leukemia models can be utilized in drug testing to verify a model that recapitulates human leukemia, to develop therapeutic "lead compounds," or to test new agents in pre-clinical studies. Fish can also serve as templates in screens of small molecule libraries or collections of existing drugs to discover compounds with unrecognized antileukemia properties. Ultimately, new knowledge of genes, pathways, or drugs arising from $D$. rerio studies requires validation in mammalian models and actual human leukemia. Due to the larger scale, more rapid turnaround, and lower cost of zebrafish studies, these models can accelerate the development of new therapies for leukemia patients. CNS central nervous system, HTS high-throughput screening, ISH in situ hybridization, IHC immunohistochemistry, LIC leukemia-initiating cell, $R G E N$ RNA-guided endonuclease, $R V s$ retroviruses, seq sequencing, SAR structure-activity relationship, TALEN transcription activator-like effector nuclease, TILLING targeting induced local lesions in genomes, $U V$ ultraviolet, $Z F N$ zinc finger nuclease

and myeloid cells such as neutrophils and monocytes [12, 13, 14•, 15•]. However, some differences between fish and mammals may be pertinent to leukemia. Notably, fish lack lymph nodes, and adult hematopoiesis occurs in 'kidney marrow,' not bone marrow. Even so, many studies have shown that genetically modified zebrafish can develop leukemias, and these $D$. rerio models can inform our understanding of human cancer.

It has now been over a decade since the first report of $\mathrm{T}$ cell ALL (T-ALL) in transgenic zebrafish [16], and in that time several other D. rerio models of T-ALL [17-20, 21••], pre-B ALL [22], AML [23], and myelopoiesis defects mimicking aspects of 'pre-AML' myeloproliferative disorders (MPDs) have been reported [24-31, 32•, 33, 34•, $35 \bullet, 36]$. Recent reviews have summarized these and many other studies, and the field is expanding rapidly [37-39, 40•, 41•, 42•, 43]. In this review, we highlight current developments in zebrafish leukemia using both genetic and xenotransplantation strategies (Fig. 1), with particular attention to exciting discoveries using $D$. rerio to probe leukemia biology, to find new drugs for these diseases, and to test existing medicines not currently used in their treatment.

\section{Techniques to Create and Investigate Zebrafish Leukemia Models}

In this section we consider methodologies used to investigate leukemia in D. rerio and describe examples of 


\section{FORWARD GENETICS}

Random DNA Modification

(UV, chemicals, RVs...)

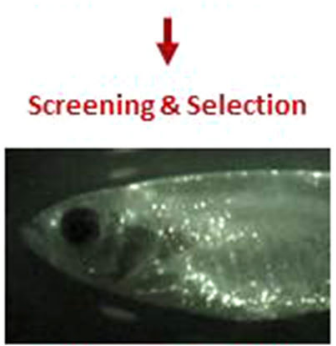

Phenotype of interest

\section{REVERSE GENETICS}

Specific Gene Modification (oncogenes/tumor-suppressors)

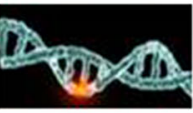

Gene of interest

- Morpholino

- mRNA Injection

- tilling

- zFN/TAlen/Rgen

- Transgenic Construct
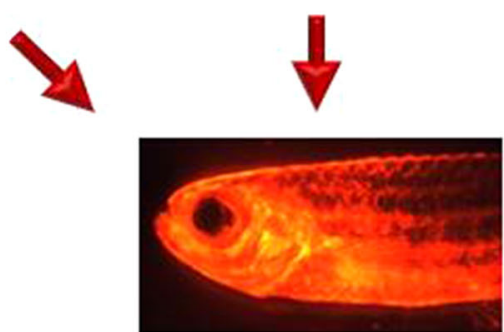

Zebrafish Leukemia Models

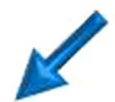

\section{GENETIC \& MOLECULAR STUDIES}

- Mutation Mapping

- Expression Profiling

- EpigeneticProfiling

- Genomic Analysis

- HTS (RNA-seq, exome seq, genome seq)

- Candidate Gene (qRT-PCR, ISH, IHC, etc.)

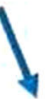

Human In vitro Studies

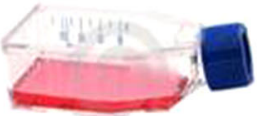

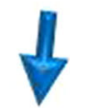

\section{IN VIVOLEUKEMIA BIOLOGY}

- Initiation

- Progression

- Dissemination (CNS invasion, Iymphoma $\rightarrow$ leukemia)

- Apoptosis Resistance Interactions
- Microenvironment

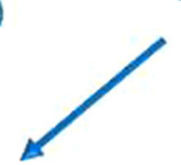

Murine Pre-Clinical Studies

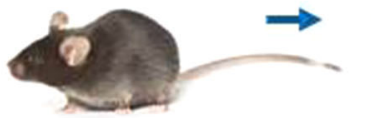

Phase II

Phase III

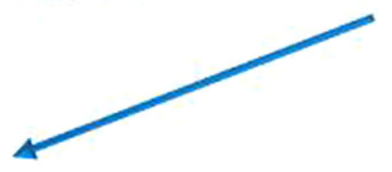

Clinical Trials

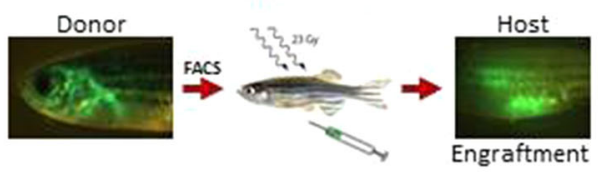

- Xeno-transplant

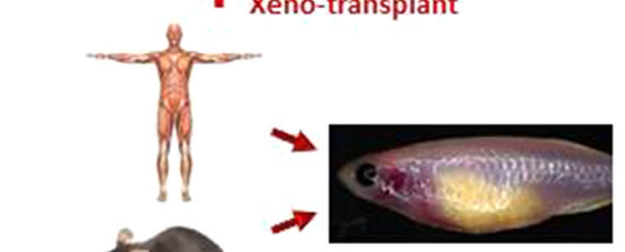

\section{LEUKEMIA-BASED}

- Allo-transplant

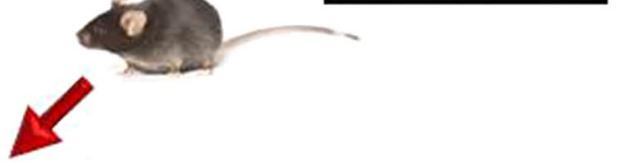

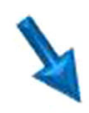

DRUG TESTING

- Model Validation

- Lead Compound Testing \& SAR

- Candidate Drug Testing

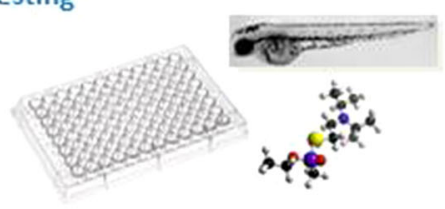

\section{DRUG SCREENING}

- Chemical Libraries

- Drug Libraries

Human Therapy

FDA Approval

Phase IV

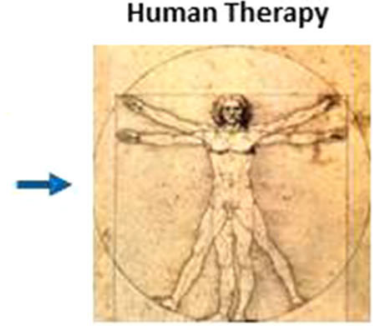


zebrafish model systems resulting from those studies. A list of leukemia models is presented in Table 1 .

\section{A Fundamental Question in Leukemia: Comprehending the Cancer Genome}

A primary objective in oncology research continues to be learning which genetic lesions cause neoplasia and gleaning mechanistic insight into how those mutations activate leukemogenesis. As in other types of cancer, recent technological advances in high-throughput sequencing (HTS; a.k.a., next-generation sequencing) have allowed for the discovery of a vast array of heterogeneous genetic mutations, some rare and insular to unique leukemia subtypes, others common and recurrent across different leukemic diseases. In human leukemias, these efforts have chiefly utilized three strategies: whole-genome sequencing, exome sequencing, and transcriptome sequencing (RNA-seq) [44]. To date, HTS of genuine leukemic genomes, exomes, and transcriptomes from $D$. rerio have not been reported, but related studies of zebrafish and human leukemic genomes and expression profiles suggest they are similar [45•, 46••].

Cancer genomics has been a subject of scrutiny for decades, and many well-defined players are household names among scientists, like the MYC, NOTCH, and RAS oncogenes and the TP53, BCL2, and PTEN tumor suppressors. Zebrafish models have investigated each of these genes in vivo, yielding findings relevant to human leukemia and other cancers [16, 19, 21••, 27, 35•, 36, 47-49]. For these proteins and other known oncogenes and tumor suppressors, functional roles were largely recognized prior to the creation of $D$. rerio models. Going forward, zebrafish's greatest utility will conceivably be in the functional analysis of newly found mutations, because HTS approaches are discovering lesions at a rate far beyond our ability to characterize them. For example, exomic HTS of 67 human T-ALLs recently revealed protein-altering mutations in over 500 genes (!) [50], and efforts in other leukemias have been similarly fruitful [44].

Obviously, it is infeasible to build $D$. rerio or other animal models for such exhaustive compendia, but zebrafish present the best opportunity to rapidly create and analyze in vivo phenotypes that derive from specific genetic mutations. Zebrafish's advantages include its rapid and ex vivo development, which favors imaging studies; its embryology and anatomy, which preserve key vertebrate features also in humans; its thoroughly annotated genome, which contains clear orthologs to at least $70 \%$ of human genes [51]; and, most importantly, its ease of genetic manipulation, particularly with regard to transgenesis. These strengths have allowed researchers to adapt $D$. rerio in a number of different ways to study leukemia.
Forward Genetic Approaches to Model Leukemia in Zebrafish

Unlike mice and most non-teleost vertebrates, zebrafish can be housed affordably in large quantities. This permits large-scale forward genetic screening projects where rare phenotypes of interest, such as leukemia, can be sought (Fig. 1). Such screens rely upon randomly modifying the $D$. rerio genome, which can be accomplished using ultraviolet light [52], chemical mutagenesis with alkylators such as $N$-ethyl- $N$-nitrosourea (ENU) [53, 54], and insertional mutagenesis using transposons or retroviral vectors [5557].

For practical reasons, forward screens usually seek early phenotypes. Thus, most are not designed to seek actual cancers, which may demand monitoring mutant fish into adulthood. Nonetheless, genes relevant to leukemia have been found by forward genetic strategies. A retroviral insertion screen discovered cancer predisposition in fish haploinsufficient for several ribosomal protein $(r p)$ genes $[55,58]$. Leukemias were not sought nor detected in this work, but human $R P$ haploinsufficiency is seen in myelodysplastic syndrome (MDS) and Diamond-Blackfan anemia, and both conditions predispose to leukemia [59]. Mutations in RPL5 and RPL10 were also recently reported in T-ALL, further implicating this class of genes [50]. In an ENU screen, the crash\&burn (crb) mutant revealed genomic stability and tumor suppressor roles for bmyb, a transcription factor [60]. Notably, translocation or duplication of human $C-M Y B$ occurs in many T-ALL cases, and murine $C-m y b$ is also a frequent site of retroviral insertions in lymphoid and myeloid leukemias [61]. Another ENU screen was cleverly designed to find genomic instability mutants via eye color in a fish strain with unique pigmentation, golden [62]. This study identified 12 genomic instability mutants with cancer predisposition [62], but like other studies, leukemias were not investigated since detecting this phenotype is problematic.

One way to circumvent this issue is to employ cellspecific markers to simplify detection of blood-borne cancers. Transgenic lines expressing green fluorescent protein (GFP) or other fluorophores exist for erythrocytes, neutrophils, immature lymphocytes, B cells, T cells, and many other lineages [14•]. We used fish with $\mathrm{T}$ cell-specific GFP [63] and ENU mutagenesis to identify $D$. rerio prone to T-ALL and created three lines with inherited predilection to this cancer [20]. However, leukemia penetrance is incomplete in these mutants, impeding efforts to identify their germline lesions. Even so, these models have been valuable in oncogenomic and drug discovery projects $\left[45^{\circ}\right.$, $64,65 \bullet, 66]$, and discerning their underlying genetic lesions remains an active area of investigation. 


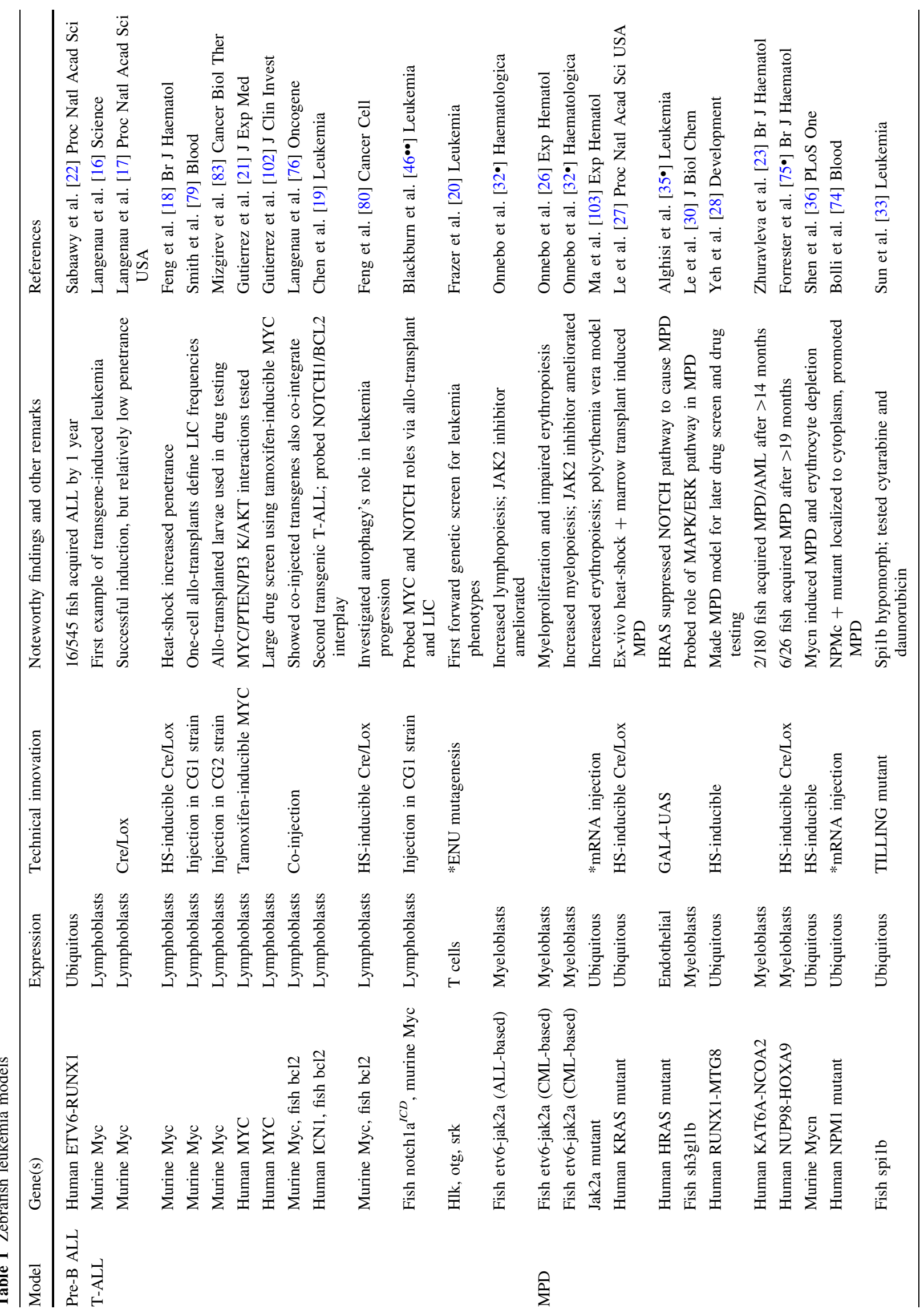


An important caveat in designing forward genetic studies is that the lesions caused by insertional mutagenesis are isolated much more easily than the single base pair changes typical of ENU approaches. This is partially offset by chemical mutagenesis' ability to theoretically induce a wider mutational spectrum than insertion events, which generally inactivate or activate an entire gene. In either case, a key strength of all forward genetic strategies is their unbiased nature and their capacity to not only create new disease models, but also to discover novel attributes of genes with no a priori evidence to suggest their role in oncogenesis.

Reverse Genetic Approaches to Model Leukemia in Zebrafish

In contrast, reverse genetic strategies require upfront knowledge about genes implicated in cancer (Fig. 1). Approaches to enhance or impede gene function in D. rerio are rapidly expanding [67] and represent the predominant strategy used by leukemia researchers. After modifying the expression or biologic activity of a candidate, the functional consequences of gene knockdown, mutation, or overexpression can then be ascertained in an in vivo context.

\section{Gene Silencing}

For years, morpholino-mediated post-transcriptional gene silencing has been the method of choice in zebrafish, and it remains a useful technique. Morpholinos are antisense oligonucleotides with a similar chemical structure to native nucleic acid. They are injected into single cell embryos, where they bind RNA to prevent protein synthesis by blocking splicing or translational initiation [68]. A major drawback of morpholinos is transiency, which limits their effect to the first several days post-fertilization (dpf). Thus, late phenotypes rarely occur. Also, because morpholinos do not modify the genome, they do not create stable lines. However, since hematopoiesis begins in early embryogenesis, knockdown by this methodology can reveal resultant expansion or contraction of blood cell lineages $[24,25,69]$.

\section{Targeted Gene Mutations}

Before techniques existed to intentionally alter $D$. rerio genes, "Targeting Induced Local Lesions IN Genomes" (TILLING) provided a means to find mutations in specific candidates. Like forward screens, TILLING employs upfront mutagenesis. Then, to locate lesions in specific genes, mutant pools are screened for base pair mismatches using a combination of PCR and nuclease digestion [70]. A recent 
application to zebrafish leukemia can be found in a study examining a TILLING-derived hypomorphic allele of spilb (pu.1), an early myelopoietic regulator. In these fish, unstable Spilb protein caused expansion of immature granulocytes as early as $3 \mathrm{dpf}$, and this persisted into adulthood where accumulation of myeloid precursors and lymphopenia mimicked aspects of MDS [33].

New techniques to specifically target $D$. rerio genes are radically altering the scope of experimental options. These methods use endonucleases to create site-specific doublestrand breaks in a gene of interest. Then, error-prone repair introduces point mutations or small insertions/deletions at the cut site. Ultimately, with crafty design and good fortune, a functional knockout or hypomorphic allele can be generated. Three related methods exist: Zinc Finger Nucleases (ZFNs), Transcription Activator-Like Effector Nucleases (TALENs), and, most recently, RNA-Guided ENdonucleases (RGENs), which are based on a bacterial CRISPR-Cas9 system [67, 71]. Unlike TILLING, which operates through multi-laboratory consortia [70], these systems allow single laboratories to scrutinize loss-offunction (LOF) phenotypes of their favorite candidate gene(s). Thus, they are particularly useful for evaluating tumor suppressors pertinent to leukemogenesis.

\section{Transgenesis}

The prevailing method to create $D$. rerio leukemia models is transgenesis (Table 1). Zebrafish readily express transgenes, and systems promoting efficient genomic integration to make stable lines are widely used $[72,73]$. Transgenes usually enact gain of function (GOF), so most studies test the effects of oncogenes. However, dominant-negative alleles can also test for leukemogenic properties of tumor suppressors with LOF. Proving high functional conservation across vertebrate species, mammalian proto-oncogenes, oncogenes with activating mutations, and fusion genes have all induced pre-leukemic and leukemic phenotypes in fish with remarkable success $[16,19,21 \bullet \cdot, 22-$ $24,28,35 \cdot, 36,74,75 \cdot$. The first of these studies coupled the $D$. rerio rag2 promoter to murine $M y c$, resulting in highly penetrant T-ALL [16]. In fact, disease was so aggressive in these fish that the line was difficult to maintain. To mitigate this, ensuing studies co-injected $\mathrm{Cre}$ recombinase or induced it by heat shock as a means to govern cancer onset $[17,18]$. Further work in this model proved co-injection of other transgenes can be a tool to alter radiation sensitivity or probe initiation in T-ALL [46••, 76]. Other projects have used $\mathrm{Cre}$-mediated expression of an activated mutant of human $K R A S^{G 12 D}$ [27] or human NUP98-HOXA9 [75•] to induce MPD in zebrafish.

Like Cre-Lox, GALA-UAS offers another tactic to conditionally express transgenes [77]. In this system, a cell- specific promoter drives transgenic yeast GALA. A second transgene of interest is flanked by a sequence, $U A S$, containing the GAL4 binding site. To unite both transgenes, the lines are bred to make double transgenics where GAL4 activates cell-specific transcription of the desired transgene. This schema was recently applied to express a GOF mutant of a different $R A S$ gene in endothelial cells carrying fli1:GAL4 [35•]. Fascinatingly, endothelial HRAS ${ }^{G 12 V}$ impaired hematopoiesis, causing myeloid differentiation arrest in marrow and accumulation of erythroid and myeloid precursors in peripheral blood. Demonstrating the power of GAL4-UAS, they also combined transgenic fli1:GAL4, UAS:HRAS ${ }^{G 12 V}$, and UAS:NICD (an active form of zebrafish notchla) to mollify the phenotype. This report is also noteworthy for its inclusion of RNA-seq from $H R A S^{G I 2 V_{-}}$and NICD-expressing larvae. Future studies with HTS of hematopoietic or overtly leukemic cells from D. rerio will be even more informative.

A final conditional expression category involves transgenes responsive to exogenous agents such as doxycycline. Such systems are powerful because adding or removing the inducing agent toggles the transgenic protein. However, because this strategy usually relies on the promoter to control expression, cell specificity is lost with standard constructs. A cunning plan to avoid this problem modulated human MYC activity in D. rerio T and T-ALL cells [21••]. Using a zebrafish rag2 promoter to enforce lymphoblast expression, an estrogen receptor [78] whose nuclear translocation is governed by tamoxifen was fused to MYC's C-terminus. In this way, fish could be housed in water \pm tamoxifen to ascertain MYC's roles in T-ALL initiation, persistence, and progression. By mixing with other transgenes or genotypes, MYC interactions with murine Akt2 or fish ptena and ptenb were also evaluated.

Of course, $D$. rerio genes themselves can also emulate human oncoproteins [26]. Again proving conserved gene function, zebrafish etv6-jak2a constructs designed from different ETV6-JAK2 fusions in human T-ALL and CML showed striking lineage fidelity [32•]. Despite using identical promoters, fish with the T-ALL fusion showed mainly lymphoid defects, while perturbed myelopoiesis occurred in fish with the CML-based construct. Clearly, vertebrate homologues' high functional preservation enables myriad strategies to engineer leukemias in D. rerio, but techniques to introduce non-endogenous leukemias into zebrafish are also gaining traction.

\section{Transplant Models of Leukemia Using Zebrafish}

Transplantions of leukemia cells between fish (allo-transplant) or from other species into D. rerio (xeno-transplant) are both useful approaches (Fig. 1). Allo-transplants are simple technically because millions of leukemic cells can 
be purified from a single donor fish, rapid intra-peritoneal injection of many recipients is feasible, and engraftment is high even with few cells. Consequently, many investigators have adopted this strategy $[16,17,19,20,22,45 \bullet, 46 \bullet \bullet, 49$, 76, 79-81]. Groups often use transplant to validate their leukemia models, as serial allo-engraftment is generally held to be an indicator of true malignancy, but more elegant allo-transplantations are also reported. For example, leukemia-initiating cell (LIC) frequencies in D. rerio T-ALL have been calculated using limiting-dilution transplants [20, 76, 81, 82]. Still, host immunosuppression by pre-irradiation tempers this assay's biologic relevance. Several studies shrewdly avoided this issue with syngeneic fish [46••, 79, 82, 83]; in one, engraftments were achieved after single cell transplants [79]! This approach has also yielded insight into the transition from pre-leukemic polyclonal expansion to outright neoplasia [46*•]. Syngeneic transplant likewise enabled in vivo chemotherapeutic testing of a serially passaged $D$. rerio T-ALL line [83].

Zebrafish that retain transparency as adults provide another valuable transplantation resource [84]. Due to their clarity, casper fish permit observations of cancer cell migration and other phenomena [85]. This feature allowed in vivo imaging of transplanted lymphoma cells as they invaded the bloodstream to become 'leukemia' [80]. Going forward, casper fish will certainly be leveraged to study other key concepts. Transplantation can also unmask genetic changes pertinent to leukemia. Sequentially passaged fish T-ALL shows higher engraftment, and hosts have shorter survival $\left[20,45^{\bullet}\right]$. Comparing de novo and derivative leukemias revealed new genomic amplifications and deletions in serially passaged cancers, and gain or loss of human homologs occur in T-ALL patients with inferior outcomes [45•]. Overall, D. rerio allo-transplantation offers tractable models for human leukemia studies.

Zebrafish also present an attractive model for xenotransplantation. In addition to the advantages cited above, embryos have limited adaptive immunity to mediate rejection, and adults can be immunosuppressed using concurrent glucocorticoids (if transplanting non-lymphoid leukemias) or pre-irradiation. Many human cancers have been xenografted into $D$. rerio (reviewed in $[43,86 \bullet, 87]$ ) to exploit these benefits, but few leukemias $[88,89]$. These studies used fish to test in vivo drug activity and are discussed later. A thorough review of zebrafish xeno- and allo-transplantation can be found elsewhere [90].

\section{Research Applications of Zebrafish Leukemia Models}

Once a leukemia model has been established, there are many ways to proceed (Fig. 1). The following sections highlight select examples of recent work interrogating
D. rerio leukemias to investigate oncogenesis and disease progression, or to discover new therapeutic agents.

\section{Classic Molecular and Cellular Biology}

Zebrafish are amenable to most tools applied to study other vertebrate cancer. While morpholinos, forward screens, and some previously cited transgenesis strategies are rather unique to $D$. rerio, standard methods such as qRT-PCR, DNA and RNA microarray, in situ hybridization (ISH), and immunohistochemistry (IHC) are employed routinely. In addition, the imaging strengths of zebrafish and the wide use of transgenic fluorophores permit many other opportunities to study leukemia cell biology in vivo.

Microarrays have compared T-ALL gene expression and acquired genomic changes between $D$. rerio and humans, showing high cross-species conservation [45•, 46••]. Functional conservation has also been explored, specifically the mechanism governing T-ALL leukemic dissemination [80]. This work showed that autophagy and focal lymphomas in fish and humans were linked to high levels of BCL2, ICAM1, and S1PR1 (sphingosine-1-phosphate receptor 1) and that genetic or pharmacologic ablation of these mediators promotes transition to T-ALL.

Classic examples of ISH, IHC, and imaging can be found in a recent paper evaluating hematopoiesis in fish with pten (a tumor suppressor) deficiency [91•]. In this study, D. rerio with combined loss of ptenalptenb displayed enhanced proliferation of stem and progenitor cells and differentiation arrest of mature blood lineages. These phenotypes were abrogated by inhibition of PI3K, a target of PTEN phosphatase.

Several studies have investigated RUNX1-MTG8 (AML1-ETO), a fusion gene frequently seen in human AML [28, 29, 31, 34•, 92]. Transgenic fish showed abnormal expression of hematopoietic regulators gatal, spil, and scl and developed MPD features [28]. Further work in this model has implicated TLE genes as AML tumor suppressors [29] and identified a leukemogenic pathway blocked by COX2 inhibition [31, 34•]. These investigators also found a benzodiazepine able to disrupt RUNX1-MTG8-induced MPD [92]. Obviously, since most of the aforementioned techniques are customary methodologies, many other groups have used similar approaches to study leukemia in D. rerio, but space constraints limit our coverage to these representative examples.

\section{Drug Testing Using Zebrafish Leukemia Models}

The ultimate goal of building and studying $D$. rerio leukemia models is to develop more effective and less toxic therapies. In an ideal cancer model, a drug active in the animal should also be efficacious in patients, and vice 
versa. In zebrafish, this premise is in its early days of validation. Vincristine and cyclophosphamide, drugs used for T-ALL treatment, were active in larvae transplanted with the $D$. rerio ZL1 T-ALL cell line, but the "gold standard" human agent prednisolone was ineffective at low-and toxic at high-concentrations [83]. However, dexamethasone, a related glucocorticoid, is known to prompt zebrafish $\mathrm{T}$ cell apoptosis [49]. In our own mostly unpublished work, dexamethasone is active against all four D. rerio T-ALL models we have tested, and we use it as a positive control when testing other new agents $[65 \bullet \cdot, 66]$. So, the same drugs are active in both species, or at least T-ALL of both species. The spil mutant MPD model was also tested using chemotherapeutics [33]. Here, cytarabine could dampen myeloproliferation, but daunorubicin showed little effect. Further testing of established drugs in these and other zebrafish models is needed to verify which compounds preserve bioactivity.

Besides known antileukemia medicines, many groups have performed assays in $D$. rerio leukemia models using pharmacologic agents not yet given to patients $[28,34 \cdot, 66$, $80,91 \cdot, 92]$. Some of these molecules are in the pipeline for eventual clinical use, while others are reagents to inhibit key pathways, acting as surrogates for drugs with similar activity not yet developed. Human leukemic xenotransplants have also been tested in fish [88, 89, 93]. Here, the biologic question is different: rather than testing agents for activity against the same type of leukemia from disparate species, the query is whether fish-based systems can provide templates for pre-clinical assays. Thus far, data with human leukemia lines are encouraging. K562 (an erythroleukemia from $B C R-A B L 1^{+}$CML) and Jurkat (a PTEN-null, NOTCH1-mutant T-ALL) were transplanted into embryos, and imatinib and cyclophosphamide responses were seen [89]. Similar findings were reported in a second study with imatinib and K562, and with all-transretinoic acid in NB4 (a PML-RARA ${ }^{+}$APML) [88], and then expanded further by testing investigational molecules against K562 in the same system [93]. If xeno-transplant can move beyond cell lines to include patient samples, "personalized medicine" assays could truly become viable.

\section{Drug Screening Using Zebrafish Leukemia Models}

D. rerio are an established platform for drug screens [9498], and their applicability to cancer-based screens is recognized [41•, 42•, 43, 99•, 100], yet no drug screens have been performed using zebrafish with genuine leukemia. This is because embryos and larvae are most practical for screens, but no $D$. rerio models manifest leukemia at such early developmental stages. Nonetheless, a handful of groups have managed to adapt drug screens in such a way as to still be pertinent to leukemia.
A famous example is the discovery that prostaglandin E2 (PGE2) promotes hematopoietic stem cell (HSC) growth [101]. This study screened $>2,300$ compounds in fish, finding several affecting PGE2 levels. Subsequent work verified PGE2's role in mammalian HSC expansion, leading to clinical testing of this medicine as a marrow recovery agent. A related body of work in RUNX1-MTG8 fish was detailed earlier [28, 31, 34•]. These investigators screened 2,000 compounds, seeking suppression of oncogene-induced changes. They found cyclooxygenase inhibitors (which block PGE synthesis) could reverse aberrant expression and that PGE2 cooperated with the transgene to induce it [31]. These projects show WT fish or fish with "pre-leukemic" phenotypes can still be highly informative.

An analogous conceptual scheme tested a $>26,000$ molecule library for agents that selectively killed normal thymocytes, with the premise that some lead compounds would be active in lymphoblastic cancers [65•*]. Secondary screening in human T-ALL lines and pre-clinical testing in D. rerio and mice validated this approach. A similar design in fish with MYC-overexpressing thymocytes was recently reported [102••]. This screen tested 4,880 molecules in larvae and a 3,194-compound library against KOPT-K1, a human T-ALL line. Ultimately, they found a group of FDA-approved drugs not presently used for leukemia treatment, identified protein phosphatase $2 \mathrm{~A}$ as their target, and defined the mechanism driving T-ALL apoptosis induced by these agents. Successful ventures like these herald the expanding role of $D$. rerio for leukemia research and ensure that such efforts will continue in the future.

\section{Conclusion: Successes and Limitations}

We have presented an overview of the growing body of work using zebrafish models to study leukemia (Fig. 1). The enlarging spectrum of techniques and the range of genetically engineered leukemias already under investigation both portend continuing success in the field. Robust models of $D$. rerio T-ALL and MPD are contributing key concepts concerning the molecular pathogenesis of these diseases. Xeno-transplantation can potentially expand our scope of inquiry considerably, as hundreds of human cell lines representing dozens of different leukemia subtypes are available. However, despite these scientific achievements, there are still many opportunities to broaden zebrafish leukemia research. Genetic models of T-ALL and MPD are well represented, but most types of human leukemia have not been studied in $D$. rerio, including the most common clinical entity, CLL. Also, only one pre-B ALL model exists, and its low incidence limits its utility. Likewise, CML is under-represented, as fish expressing $B C R-A B L 1$ have not been created. Finally, although several 
informative MPD models are reported, these rarely progress to an equivalent of human AML. In the future, these challenges will undoubtedly be vanquished by clever scientists using innovative strategies. In the interim, much work remains with existing models, as our knowledge of the causal pathways in leukemogenesis is far from comprehensive. Encouragingly, even without cognizance of the precise mechanisms responsible, we are using zebrafish to procure an abundance of tantalizing therapeutic leads. Testing of promising drugs in pre-clinical settings and screening for novel agents and targetable pathways are both ongoing, proving $D$. rerio can advance treatments for these cancers. Going forward, zebrafish will continue to provide valuable options for translational projects as we endeavor to conquer these diseases.

\section{Compliance with Ethics Guidelines}

Conflict of Interest Barbara Squiban declares no conflict of interest. J. Kimble Frazer has received grants from CureSearch for Children's Cancer and has spoken at the 6th Aquatic Animal Models for Human Disease meeting in Milwaukee, WI, outside of the submitted work. Dr. Frazer has also been supported by a K08 award from NIH/NICHD and a COBRE award from NIH/NIGMS.

Human and Animal Rights and Informed Consent This article does not contain any studies with human or animal subjects performed by any of the authors.

\section{References}

Papers of particular importance have been highlighted as:

- Of importance

-• Of major importance

1. Common Cancer Types (2013) http://www.cancer.gov/ cancertopics/types/commoncancers National Cancer Institute of the National Institutes of Health

2. Cancer incidence for common cancers (2013) http://www. cancerresearchuk.org/cancer-info/cancerstats/incidence/common cancers/\#Twenty: Cancer Research UK

3. SEER database: Leukemia (2013) http://seer.cancer.gov/statfacts/ html/leuks.html

4. Pui CH, Evans WE (2006) Treatment of acute lymphoblastic leukemia. N Engl J Med 354:166-178

5. Druker BJ, Sawyers CL, Kantarjian H, Resta DJ, Reese SF et al (2001) Activity of a specific inhibitor of the BCR-ABL tyrosine kinase in the blast crisis of chronic myeloid leukemia and acute lymphoblastic leukemia with the Philadelphia chromosome. N Engl J Med 344:1038-1042

6. Druker BJ, Talpaz M, Resta DJ, Peng B, Buchdunger E et al (2001) Efficacy and safety of a specific inhibitor of the BCRABL tyrosine kinase in chronic myeloid leukemia. N Engl $\mathrm{J}$ Med 344:1031-1037

7. Kirienko NV, Mani K, Fay DS (2010) Cancer models in Caenorhabditis elegans. Dev Dyn 239:1413-1448

8. Crozatier M, Vincent A (2011) Drosophila: a model for studying genetic and molecular aspects of haematopoiesis and associated leukaemias. Dis Model Mech 4:439-445
9. Rudrapatna VA, Cagan RL, Das TK (2012) Drosophila cancer models. Dev Dyn 241:107-118

10. Gonzalez C (2013) Drosophila melanogaster: a model and a tool to investigate malignancy and identify new therapeutics. Nat Rev Cancer 13:172-183

11. Wang L, Kounatidis I, Ligoxygakis P (2014) Drosophila as a model to study the role of blood cells in inflammation, innate immunity and cancer. Front Cell Infect Microbiol 3:113

12. Meeker ND, Trede NS (2008) Immunology and zebrafish: spawning new models of human disease. Dev Comp Immunol 32:745-757

13. Lieschke GJ, Trede NS (2009) Fish immunology. Curr Biol 19:R678-R682

14. - Stachura DL, Traver D (2011) Cellular dissection of zebrafish hematopoiesis. Methods Cell Biol 101:75-110. Comprehensive review of $D$. rerio hematopoiesis and techniques to study these models

15. - Renshaw SA, Trede NS (2012) A model 450 million years in the making: zebrafish and vertebrate immunity. Dis Model Mech 5:38-47. Concise review of zebrafish immunology and its similarities and differences to mammals

16. Langenau DM, Traver D, Ferrando AA, Kutok JL, Aster JC et al (2003) Myc-induced $\mathrm{T}$ cell leukemia in transgenic zebrafish. Science 299:887-890

17. Langenau DM, Feng H, Berghmans S, Kanki JP, Kutok JL et al (2005) Cre/lox-regulated transgenic zebrafish model with conditional myc-induced T cell acute lymphoblastic leukemia. Proc Natl Acad Sci USA 102:6068-6073

18. Feng H, Langenau DM, Madge JA, Quinkertz A, Gutierrez A et al (2007) Heat-shock induction of T-cell lymphoma/leukaemia in conditional Cre/lox-regulated transgenic zebrafish. Br J Haematol 138:169-175

19. Chen J, Jette C, Kanki JP, Aster JC, Look AT et al (2007) NOTCH1-induced T-cell leukemia in transgenic zebrafish. Leukemia 21:462-471

20. Frazer JK, Meeker ND, Rudner L, Bradley DF, Smith AC et al (2009) Heritable T-cell malignancy models established in a zebrafish phenotypic screen. Leukemia 23:1825-1835

21. • Gutierrez A, Grebliunaite R, Feng H, Kozakewich E, Zhu S, et al. (2011) Pten mediates Myc oncogene dependence in a conditional zebrafish model of $\mathrm{T}$ cell acute lymphoblastic leukemia. J Exp Med 208:1595-1603. Created transgenic fish with tamoxifen-induced conditionally active human MYC to probe interactions between MYC, PTEN, and AKT. Revealed that PTEN LOF or AKT GOF render T-ALL MYC-independent. Found MYC necessary for leukemia initiation, but not progression

22. Sabaawy HE, Azuma M, Embree LJ, Tsai HJ, Starost MF et al (2006) TEL-AML1 transgenic zebrafish model of precursor B cell acute lymphoblastic leukemia. Proc Natl Acad Sci USA 103:15166-15171

23. Zhuravleva J, Paggetti J, Martin L, Hammann A, Solary E et al (2008) MOZ/TIF2-induced acute myeloid leukaemia in transgenic fish. Br J Haematol 143:378-382

24. Kalev-Zylinska ML, Horsfield JA, Flores MV, Postlethwait JH, Vitas MR et al (2002) Runx1 is required for zebrafish blood and vessel development and expression of a human RUNX1CBF2T1 transgene advances a model for studies of leukemogenesis. Development 129:2015-2030

25. Kalev-Zylinska ML, Horsfield JA, Flores MV, Postlethwait JH, Chau JY et al (2003) Runx3 is required for hematopoietic development in zebrafish. Dev Dyn 228:323-336

26. Onnebo SM, Condron MM, McPhee DO, Lieschke GJ, Ward AC (2005) Hematopoietic perturbation in zebrafish expressing a tel-jak2a fusion. Exp Hematol 33:182-188

27. Le X, Langenau DM, Keefe MD, Kutok JL, Neuberg DS et al (2007) Heat shock-inducible Cre/Lox approaches to induce 
diverse types of tumors and hyperplasia in transgenic zebrafish. Proc Natl Acad Sci USA 104:9410-9415

28. Yeh JR, Munson KM, Chao YL, Peterson QP, Macrae CA et al (2008) AML1-ETO reprograms hematopoietic cell fate by downregulating scl expression. Development 135:401-410

29. Dayyani F, Wang J, Yeh JR, Ahn EY, Tobey E et al (2008) Loss of TLE1 and TLE4 from the del(9q) commonly deleted region in AML cooperates with AML1-ETO to affect myeloid cell proliferation and survival. Blood 111:4338-4347

30. Le HY, Zhang Y, Liu H, Ma LH, Jin Y et al (2008) eena Promotes myeloid proliferation through stimulating ERK1/2 phosphorylation in zebrafish. J Biol Chem 283:17652-17661

31. Yeh JR, Munson KM, Elagib KE, Goldfarb AN, Sweetser DA et al (2009) Discovering chemical modifiers of oncogeneregulated hematopoietic differentiation. Nat Chem Biol 5: 236-243

32. - Onnebo SM, Rasighaemi P, Kumar J, Liongue C, Ward AC (2012) Alternative TEL-JAK2 fusions associated with T-cell acute lymphoblastic leukemia and atypical chronic myelogenous leukemia dissected in zebrafish. Haematologica 97:1895-1903. Compared two versions of ETV6-JAK2 fusion from human T-ALL and CML cases; transgenic fish showed lineage-specific phenotypes

33. Sun J, Liu W, Li L, Chen J, Wu M et al (2013) Suppression of Pu.1 function results in expanded myelopoiesis in zebrafish. Leukemia 27:1913-1917

34. - Zhang Y, Wang J, Wheat J, Chen X, Jin S, et al. (2013) AML1-ETO mediates hematopoietic self-renewal and leukemogenesis through a COX/beta-catenin signaling pathway. Blood 121:4906-4916. One of a series of reports based on findings from a drug screen in RUNX1-MTG8 transgenic fish that previously implicated cyclooxygenase and $\beta$-catenin in MPD/AML leukemogenesis

35. - Alghisi E, Distel M, Malagola M, Anelli V, Santoriello C, et al. (2013) Targeting oncogene expression to endothelial cells induces proliferation of the myelo-erythroid lineage by repressing the Notch pathway. Leukemia 27:2229-2241. Used transgenic HRAS $S^{G 12 V}$ mutant to induce MPD, where RAS suppresses $\mathrm{NOTCH}$

36. Shen LJ, Chen FY, Zhang Y, Cao LF, Kuang Y et al (2013) MYCN transgenic zebrafish model with the characterization of acute myeloid leukemia and altered hematopoiesis. PLoS One 8:e59070

37. Stoletov K, Klemke R (2008) Catch of the day: zebrafish as a human cancer model. Oncogene 27:4509-4520

38. Payne E, Look T (2009) Zebrafish modelling of leukaemias. Br J Haematol 146:247-256

39. Mione MC, Trede NS (2010) The zebrafish as a model for cancer. Dis Model Mech 3:517-523

40. • Etchin J, Kanki JP, Look AT (2011) Zebrafish as a model for the study of human cancer. Methods Cell Biol 105:309-337. Comprehensive review of D. rerio models of leukemia and other human cancers, including applications of these models

41. • Teittinen KJ, Gronroos T, Parikka M, Ramet M, Lohi O (2012) The zebrafish as a tool in leukemia research. Leuk Res 36:1082-1088. Another recent review of zebrafish leukemia research

42. - White R, Rose K, Zon L (2013) Zebrafish cancer: the state of the art and the path forward. Nat Rev Cancer 13:624-636. Very recent overview of zebrafish cancer models and strategies for their analysis

43. Mimeault M, Batra SK (2013) Emergence of zebrafish models in oncology for validating novel anticancer drug targets and nanomaterials. Drug Discov Today 18:128-140

44. Mullighan CG (2013) Genome sequencing of lymphoid malignancies. Blood 122:3899-3907
45. - Rudner LA, Brown KH, Dobrinski KP, Bradley DF, Garcia MI, et al. (2011) Shared acquired genomic changes in zebrafish and human T-ALL. Oncogene 30:4289-4296. Used array comparative genomic hybridization of D. rerio T-ALL to find amplified and deleted genes in both human and fish T-ALL

46. • Blackburn JS, Liu S, Raiser DM, Martinez SA, Feng H, et al. (2012) Notch signaling expands a pre-malignant pool of T-cell acute lymphoblastic leukemia clones without affecting leukemia-propagating cell frequency. Leukemia 26:2069-2078. Investigated the interplay of NOTCH and MYC in T-ALL. Found NOTCH1 GOF increased pre-malignant $T$ cells, but did not increase LIC frequency. Cross-species expression profiling revealed pathway conservation in human and fish $T-A L L$

47. Berghmans S, Murphey RD, Wienholds E, Neuberg D, Kutok JL et al (2005) tp53 mutant zebrafish develop malignant peripheral nerve sheath tumors. Proc Natl Acad Sci USA 102:407-412

48. Parant JM, George SA, Holden JA, Yost HJ (2010) Genetic modeling of Li-Fraumeni syndrome in zebrafish. Dis Model Mech 3:45-56

49. Langenau DM, Jette C, Berghmans S, Palomero T, Kanki JP et al (2005) Suppression of apoptosis by bcl-2 overexpression in lymphoid cells of transgenic zebrafish. Blood 105:3278-3285

50. De Keersmaecker K, Atak ZK, Li N, Vicente C, Patchett S et al (2013) Exome sequencing identifies mutation in CNOT3 and ribosomal genes RPL5 and RPL10 in T-cell acute lymphoblastic leukemia. Nat Genet 45:186-190

51. Howe K, Clark MD, Torroja CF, Torrance J, Berthelot C et al (2013) The zebrafish reference genome sequence and its relationship to the human genome. Nature 496:498-503

52. Grunwald DJ, Streisinger G (1992) Induction of mutations in the zebrafish with ultraviolet light. Genet Res 59:93-101

53. Grunwald DJ, Streisinger G (1992) Induction of recessive lethal and specific locus mutations in the zebrafish with ethyl nitrosourea. Genet Res 59:103-116

54. Solnica-Krezel L, Schier AF, Driever W (1994) Efficient recovery of ENU-induced mutations from the zebrafish germline. Genetics 136:1401-1420

55. Amsterdam A, Sadler KC, Lai K, Farrington S, Bronson RT et al (2004) Many ribosomal protein genes are cancer genes in zebrafish. PLoS Biol 2:E139

56. Amsterdam A, Varshney GK, Burgess SM (2011) Retroviralmediated Insertional Mutagenesis in Zebrafish. Methods Cell Biol 104:59-82

57. Varshney GK, Lu J, Gildea DE, Huang H, Pei W et al (2013) A large-scale zebrafish gene knockout resource for the genomewide study of gene function. Genome Res 23:727-735

58. MacInnes AW, Amsterdam A, Whittaker CA, Hopkins N, Lees JA (2008) Loss of p53 synthesis in zebrafish tumors with ribosomal protein gene mutations. Proc Natl Acad Sci USA 105:10408-10413

59. Liu S, Leach SD (2011) Zebrafish models for cancer. Annu Rev Pathol 6:71-93

60. Shepard JL, Amatruda JF, Stern HM, Subramanian A, Finkelstein D et al (2005) A zebrafish bmyb mutation causes genome instability and increased cancer susceptibility. Proc Natl Acad Sci USA 102:13194-13199

61. Clappier E, Cuccuini W, Kalota A, Crinquette A, Cayuela JM et al (2007) The C-MYB locus is involved in chromosomal translocation and genomic duplications in human T-cell acute leukemia (T-ALL), the translocation defining a new T-ALL subtype in very young children. Blood 110:1251-1261

62. Moore JL, Rush LM, Breneman C, Mohideen MA, Cheng KC (2006) Zebrafish genomic instability mutants and cancer susceptibility. Genetics 174:585-600

63. Langenau DM, Ferrando AA, Traver D, Kutok JL, Hezel JP et al (2004) In vivo tracking of $T$ cell development, ablation, and 
engraftment in transgenic zebrafish. Proc Natl Acad Sci USA 101:7369-7374

64. Frazer JK, Batchelor LA, Bradley DF, Brown KH, Dobrinski KP et al (2012) Genomic amplification of an endogenous retrovirus in zebrafish T-cell malignancies. Adv Hematol 2012:627920

65. • Ridges S, Heaton WL, Joshi D, Choi H, Eiring A, et al. (2012) Zebrafish screen identifies novel compound with selective toxicity against leukemia. Blood 119:5621-5631. Largest small molecule screen in fish to find anti-leukemic compounds. Identified a novel agent that kills fish thymocytes and T-ALL cell lines. Showed efficacy in both a fish T-ALL model and murine xenografts

66. Christoph S, Deryckere D, Schlegel J, Frazer JK, Batchelor LA et al (2013) UNC569, a novel small-molecule mer inhibitor with efficacy against acute lymphoblastic leukemia in vitro and in vivo. Mol Cancer Ther 12:2367-2377

67. Huang P, Zhu Z, Lin S, Zhang B (2012) Reverse genetic approaches in zebrafish. J Genet Genomics 39:421-433

68. Heasman J (2002) Morpholino oligos: making sense of antisense? Dev Biol 243:209-214

69. Fu CT, Zhu KY, Mi JQ, Liu YF, Murray ST et al (2010) An evolutionarily conserved PTEN-C/EBPalpha-CTNNA1 axis controls myeloid development and transformation. Blood 115:4715-4724

70. Moens CB, Donn TM, Wolf-Saxon ER, Ma TP (2008) Reverse genetics in zebrafish by TILLING. Brief Funct Genomic Proteomic 7:454-459

71. Hwang WY, Fu Y, Reyon D, Maeder ML, Tsai SQ et al (2013) Efficient genome editing in zebrafish using a CRISPR-Cas system. Nat Biotechnol 31:227-229

72. Kwan KM, Fujimoto E, Grabher C, Mangum BD, Hardy ME et al (2007) The Tol2kit: a multisite gateway-based construction kit for Tol2 transposon transgenesis constructs. Dev Dyn 236:3088-3099

73. Suster ML, Kikuta H, Urasaki A, Asakawa K, Kawakami K (2009) Transgenesis in zebrafish with the tol 2 transposon system. Methods Mol Biol 561:41-63

74. Bolli N, Payne EM, Grabher C, Lee JS, Johnston AB et al (2010) Expression of the cytoplasmic NPM1 mutant $(\mathrm{NPMc}+)$ causes the expansion of hematopoietic cells in zebrafish. Blood 115:3329-3340

75. - Forrester AM, Grabher C, McBride ER, Boyd ER, Vigerstad MH, et al. (2011) NUP98-HOXA9-transgenic zebrafish develop a myeloproliferative neoplasm and provide new insight into mechanisms of myeloid leukaemogenesis. $\mathrm{Br} \mathrm{J}$ Haematol 155:167-181. Created NUP98-HOXA9 transgenic model of MPD, where $\sim 25 \%$ of fish develop disease beyond 18 months

76. Langenau DM, Keefe MD, Storer NY, Jette CA, Smith AC et al (2008) Co-injection strategies to modify radiation sensitivity and tumor initiation in transgenic Zebrafish. Oncogene 27:4242-4248

77. Scheer N, Campos-Ortega JA (1999) Use of the Gal4-UAS technique for targeted gene expression in the zebrafish. Mech Dev 80:153-158

78. Littlewood TD, Hancock DC, Danielian PS, Parker MG, Evan GI (1995) A modified oestrogen receptor ligand-binding domain as an improved switch for the regulation of heterologous proteins. Nucleic Acids Res 23:1686-1690

79. Smith AC, Raimondi AR, Salthouse CD, Ignatius MS, Blackburn JS et al (2010) High-throughput cell transplantation establishes that tumor-initiating cells are abundant in zebrafish T-cell acute lymphoblastic leukemia. Blood 115:3296-3303

80. Feng H, Stachura DL, White RM, Gutierrez A, Zhang L et al (2010) T-lymphoblastic lymphoma cells express high levels of BCL2, S1P1, and ICAM1, leading to a blockade of tumor cell intravasation. Cancer Cell 18:353-366
81. Ignatius MS, Langenau DM (2009) Zebrafish as a model for cancer self-renewal. Zebrafish 6:377-387

82. Blackburn JS, Liu S, Langenau DM (2011) Quantifying the frequency of tumor-propagating cells using limiting dilution cell transplantation in syngeneic zebrafish. J Vis Exp 14:e2790

83. Mizgirev IV, Revskoy S (2010) A new zebrafish model for experimental leukemia therapy. Cancer Biol Ther 9:895-902

84. White RM, Sessa A, Burke C, Bowman T, LeBlanc J et al (2008) Transparent adult zebrafish as a tool for in vivo transplantation analysis. Cell Stem Cell 2:183-189

85. Pugach EK, Li P, White R, Zon L (2009) Retro-orbital injection in adult zebrafish. J Vis Exp

86. - Konantz M, Balci TB, Hartwig UF, Dellaire G, Andre MC, et al. (2012) Zebrafish xenografts as a tool for in vivo studies on human cancer. Ann N Y Acad Sci 1266:124-137. Review of xeno-transplant strategies using $D$. rerio to study human cancers

87. Tobia C, Gariano G, De Sena G, Presta M (2013) Zebrafish embryo as a tool to study tumor/endothelial cell cross-talk. Biochim Biophys Acta 1832:1371-1377

88. Corkery DP, Dellaire G, Berman JN (2011) Leukaemia xenotransplantation in zebrafish-chemotherapy response assay in vivo. Br J Haematol 153:786-789

89. Pruvot B, Jacquel A, Droin N, Auberger P, Bouscary D et al (2011) Leukemic cell xenograft in zebrafish embryo for investigating drug efficacy. Haematologica 96:612-616

90. Taylor AM, Zon LI (2009) Zebrafish tumor assays: the state of transplantation. Zebrafish 6:339-346

91. - Choorapoikayil S, Kers R, Herbomel P, Kissa K, den Hertog J (2014) Pivotal role of Pten in the balance between proliferation and differentiation of hematopoietic stem cells in zebrafish. Blood 123:184-190. Showed PTEN LOF causes hematopoietic stem/precursor cell hyper-proliferation with arrested differentiation of mature blood lineages; PI3K inhibition attenuated the phenotype

92. Cunningham L, Finckbeiner S, Hyde RK, Southall N, Marugan J et al (2012) Identification of benzodiazepine Ro5-3335 as an inhibitor of CBF leukemia through quantitative high throughput screen against RUNX1-CBFbeta interaction. Proc Natl Acad Sci USA 109:14592-14597

93. Smithen DA, Forrester AM, Corkery DP, Dellaire G, Colpitts J et al (2013) Investigations regarding the utility of prodigiosenes to treat leukemia. Org Biomol Chem 11:62-68

94. Ali S, Champagne DL, Spaink HP, Richardson MK (2011) Zebrafish embryos and larvae: a new generation of disease models and drug screens. Birth Defects Res C Embryo Today 93:115-133

95. Delvecchio C, Tiefenbach J, Krause HM (2011) The zebrafish: a powerful platform for in vivo, HTS drug discovery. Assay Drug Dev Technol 9:354-361

96. Lessman CA (2011) The developing zebrafish (Danio rerio): a vertebrate model for high-throughput screening of chemical libraries. Birth Defects Res C Embryo Today 93:268-280

97. Peterson RT, Fishman MC (2011) Designing zebrafish chemical screens. Methods Cell Biol 105:525-541

98. Peterson RT, Macrae CA (2012) Systematic approaches to toxicology in the zebrafish. Annu Rev Pharmacol Toxicol 52:433-453

99. - Zhang Y, Yeh JR (2012) In vivo chemical screening for modulators of hematopoiesis and hematological diseases. Adv Hematol 2012: 851674. Recent review focused on hematopoiesis- and leukemia-relevant zebrafish in vivo drug screening

100. Terriente J, Pujades C (2013) Use of zebrafish embryos for small molecule screening related to cancer. Dev Dyn 242:97-107 
101. North TE, Goessling W, Walkley CR, Lengerke C, Kopani KR et al (2007) Prostaglandin E2 regulates vertebrate haematopoietic stem cell homeostasis. Nature 447:1007-1011

102. • Gutierrez A, Pan L, Groen RW, Baleydier F, Kentsis A, et al. (2014) Phenothiazines induce PP2A-mediated apoptosis in T cell acute lymphoblastic leukemia. J Clin Invest 124:644-655. Used two drug screens to identify PP2A phosphatase as a T-ALL drug target. One screen tested larvae with transgenic MYC in T cells; another sought agents potentiating $\gamma$-secretase inhibition in a T-ALL line. Found that phenothiazines stimulate PP2Amediated apoptosis

103. Ma AC, Fan A, Ward AC, Liongue C, Lewis RS et al (2009) A novel zebrafish jak2a(V581F) model shared features of human JAK2(V617F) polycythemia vera. Exp Hematol 37(1379-1386): e1374 